\title{
An integrated system for managing multidisciplinary oceanographic data collected in the Mediterranean Sea during the basin-scale research project EU/MAST-MATER (1996-2000)
}

C. Maillard ${ }^{a}, *$ E. Balopoulos ${ }^{b}$, A. Giorgetti ${ }^{c}$, M. Fichaut ${ }^{a}, A$. Iona $^{b}$, M. Larour ${ }^{d}$, A. Latrouite $^{a}$, B. Manca $^{c}$, G. Maudire ${ }^{d}$, P. Nicolas ${ }^{e}$ and J. -A. Sanchez-Cabeza ${ }^{\dagger}$

${ }^{a}$ SISMER, BP 70, 29280 Plouzane, France

${ }^{\mathrm{b}} \mathrm{NCMR/HNODC}$, Hellinikon, 16604 Athens, Greece

c OGS, PO Box 2011, 34016 Trieste, Italy

d IFREMER/ISIB, BP 70, 29280 Plouzane, France

${ }^{e}$ SAFEGE/CETIIS, 30 Av. Malacrida, 13100 Aix en Provence, France

${ }^{f}$ Universitat Autonoma de Barcelona, 08193 Bellaterra, Spain

*: Corresponding author : Tel.: +33-2-98-22-42-79; fax: +33-2-98-22-46-44; email: Catherine.Maillard@ifremer.fr

\begin{abstract}
:
An advanced computer and communication technology was used to develop an integrated system and software tools for managing a great diversity of oceanographic data collected in the Mediterranean Sea during 1996-2000. Data were obtained during 108 sea cruises, carried out within the framework of the large-scale international research project MATER (mass transfer and ecosystem response), which was financially supported by the Marine Science and Technology (MAST) Programme of the European Union (EU). Data collection involved the active participation of various research vessels and personnel coming from 58 different laboratories of 13 countries. Data formatting as well as automatic and visual data quality controls were implemented using internationally accepted standards and procedures. Various data inventories and meta-data information, accessible through the World Wide Web (WWW), are made available to the user community. A database was developed, which, along with meta-data and other data relevant to the project information, is made available to the user community in the form of a CD-ROM. The database consists of 5861 vertical profiles and 842 time series of basic physical and biogeochemical parameters collected in the seawater column as well as biogeochemical parameters from the analysis of 70 sediment cores. Furthermore, it includes 67 cruise data files of nonstandard additional biological and atmospheric parameters.
\end{abstract}

Keywords: Mediterranean Sea; Ocean data; Data management; Quality assurance; Data product 


\section{Introduction}

The Mediterranean Sea has been the subject of an intensive investigation, especially during the last decade, when the Mediterranean Targeted Projects (MTP-I \& MTP-II) were launched by the Marine Science and Technology (MAST) Programme of the European Union. The EU/MAST-MATER research Project, aiming at studying "Mass Transfer and Ecosystem Response" of the Mediterranean Sea has been a major multidisciplinary fieldwork. It was implemented during the period 1996-2000 and gathered 58 research groups from 10 EU Member States and 3 non-EU States. During the Project implementation, a total of 108 oceanographic cruises (representing more than 1000 days of ship time) were carried out by 12 research vessels (coming from 6 different countries) and about 254 main scientific equipment was used. This resulted in the collection of a data set consisting of a large amount of a great diversity of various measured parameters.

To achieve a harmonized data management, within the EU/MAST-MATER Project, the "code on data management" issued by EU/MAST (http://www.sea-search.net/guidelines-practices/) was adopted. According to this, meta-data information (e.g., cruise, mooring and data sets summaries) should be made available shortly after the cruise implementation, and the data should be made available one year after the end of the project. Due to the large number of parameters measured by the various research groups participating in the Project, it was necessary not only to get and circulate the meta-data and the data, but also to check the coherence and the compatibility of the different data sets and to prepare a final integrated data product. This has been made possible by developing a qualified data management structure, a common protocol for data formatting and checking and finally appropriate software tools to insure timely and standardised implementation of the data management tasks.

In this contribution, the various elements of the data management plan within the EU/MAST-MATER Project are presented and the resulting MTPII-MATER Database CD-ROM (Mater Group, 2001) is described.

\section{Data Management Structure and Data Flow}

A distributed data management structure, based on three regional data centres was designed according to the scheme presented in Fig.1. Each of them had the responsibility for data management in particular region of the Mediterranean Sea. The selection of the data centres was made taking into account that the fieldwork activity of the Project was, for practical reasons, organized on the basis of three major regions of the Mediterranean Sea, that is the Eastern, Central and Western Mediterranean regions. It was also taken into consideration that the major data collecting laboratories in the aforementioned regions were Hellenic, Italian, Spanish and French. 


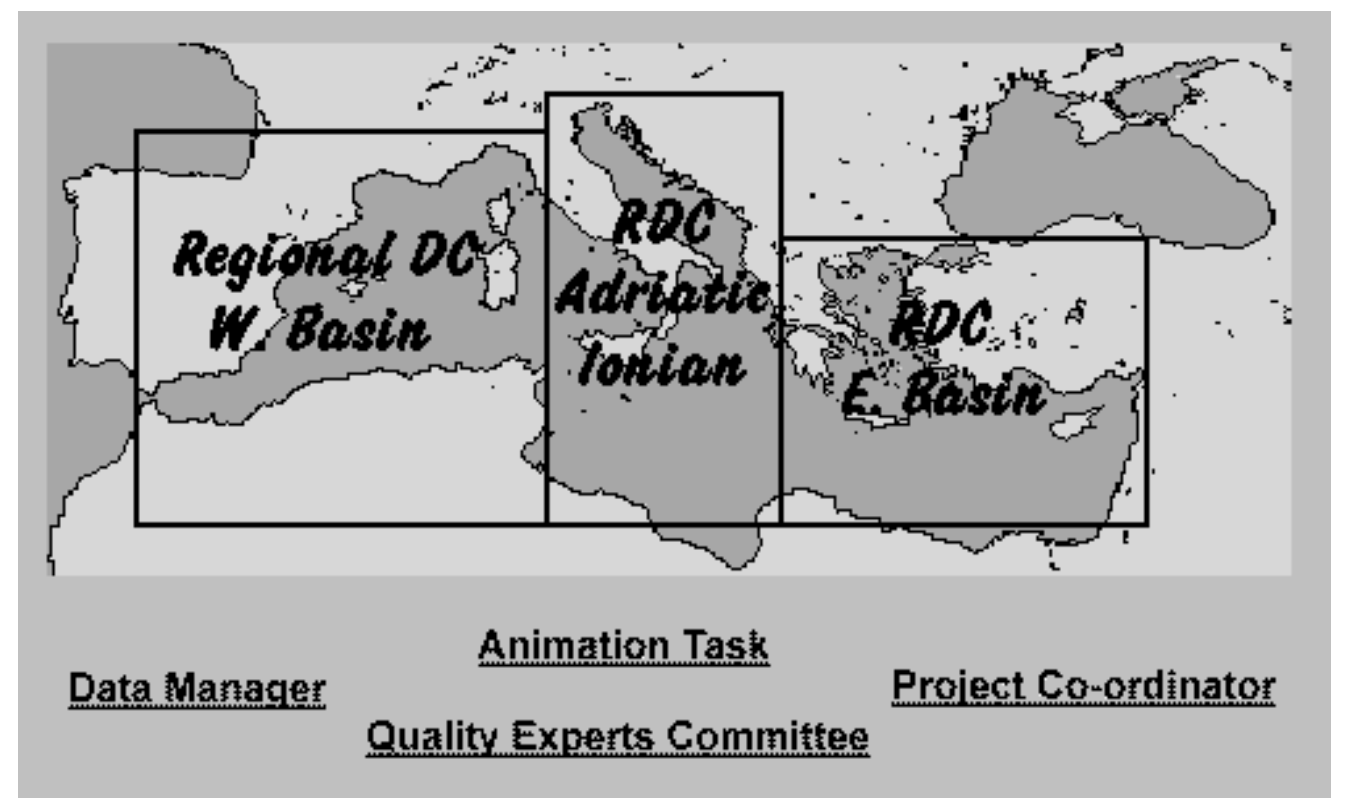

Fig. 1. MTPII-MATER data management structure

The three data centres selected and the regions of their responsibility are as follows:

(a) National Centre for Marine Research, Hellenic National Oceanographic Data Centre (NCMR/HNODC, Greece): Regional Data Centre for the Eastern Mediterranean Sea

(b) Istituto Nazionale di Oceanografia e di Geofisica Sperimentale - OGS, Italy: Regional Data Centre for the Central Mediterranean Sea (Adriatic, Ionian Seas, Sicily Straits and Sardinia Channel)

(c) Institut Français de Recherche pour l'Explotation de la MER (IFREMER/SISMER, France): Coordinating data Centre and responsible for the Western Mediterranean Sea.

The project data management was supported by a Data Quality Expert Group (DQEG) (chaired by the Project co-ordinator) with the aim of supervising and reviewing data collection techniques and also samples analyses methodologies, if necessary. In addition, the Project management unit contributed to the Project data management maintaining information on the schedule of the various oceanographic vessels involved in data collection and synthesis of the field work (Animation Task).

The organizational scheme for meta-data and data flow included the following steps:

- Search for cruise schedule, both from project and other national sources

- Request meta-data, including summary reports for cruises (ROSCOP), moorings, instruments, data sets (EDMED), by sending the appropriate forms to the data collecting groups.

- Request data participating in the Project laboratories, on the basis of the summary reports received by the data centres.

- Reformat, safeguard, check for quality, report to the source scientist(s) and eventually ask for further evaluation or advise to the Data Quality Expert Group

- Publish up-to-date catalogues of data and meta-data on World Wide Web (WWW) servers to insure a permanent visibility of the status of the data to the project consortium

- Disseminate data and meta-data according to the project policy (with author permission and restricted to the partners during the project implementation). 
This scheme was rather complex, reflecting the complexity of the project itself; however the main links of data flow have been between the scientific laboratories and the data centres.

\section{Inventories and Documentation}

To facilitate meta-data and data flow between the Project participating scientists, four regional inventories were developed and continuously updated by the three regional data centres. These inventories were related to the Project cruises, moorings, instruments and data sets, respectively.

The "Cruises Reports" inventory provides information on the cruises carried out in each of the abovementioned regions of the Mediterranean Sea, listed by year. Clicking on any of the cruise, returns to the corresponding cruise report, which includes stations position (example in Fig.2), list of collected data and list of archived data (with location). This inventory, including the coded information on observations made during the cruise (ROSCOP parameters), has been a key tool, for the overall management activity, which was used to request data of collecting scientific groups.

The "Moorings Reports" inventory includes a list and summary reports of the 130 moorings or experiments carried out with the release of Lagrangian drifters. It completes the cruises summaries inventory for data collected automatically between the cruises.

The "Instruments Reports" inventory includes a list and brief description of the 254 main instruments used during the project, the owner laboratories and information on the sensors calibrations, when available. 


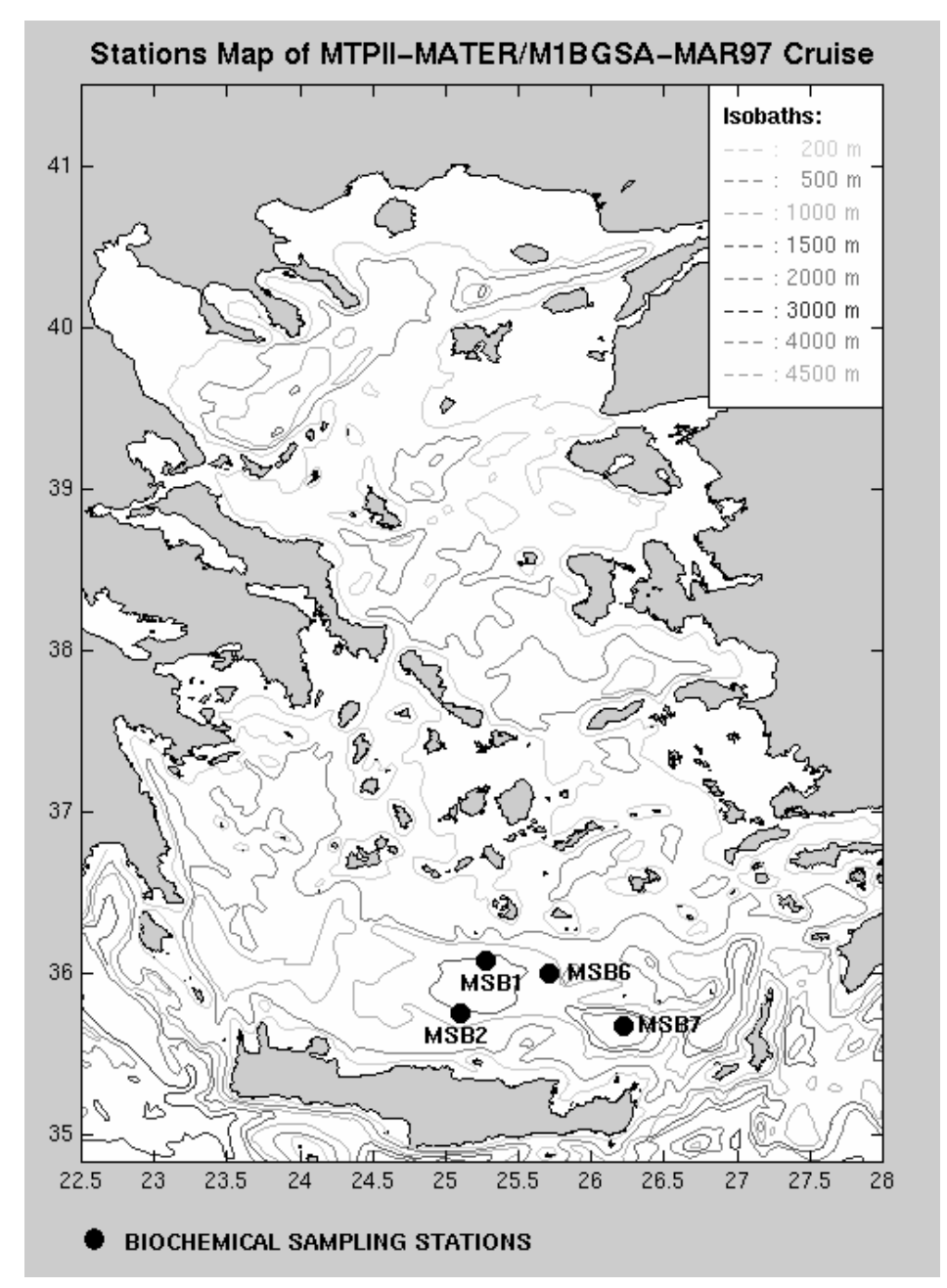

Fig. 2. Example of cruise track reported in the summary cruise reports WWW catalog

The "Data sets Reports" inventory includes a list, linked to the general description of the main data types collected at the European EDMED format (Euronodim Group, 2000) with information on sensors, methods, observed parameters and area covered. The general descriptions are linked with a detailed descriptions (location and date) of each data file that consists in the data of the same type collected during the same cruise. The list of the data sets described in this catalogue is provided in Table I.

\begin{tabular}{|l|l|}
\hline Physics & ADCP current measurements (W-Mediterranean) \\
\cline { 2 - 3 } & ADCP Eulerian Current Time Series (Adriatic/lonian Sea) \\
\cline { 2 - 3 } & CTD profiles (W-Mediterranean) \\
\hline & CTD Profiles (Adriatic/lonian Sea) \\
\hline & CTD Profiles (Sicily/Sardinia/Tunisia Region) \\
\hline & CTD Profiles (E-Mediterranean) \\
\hline & Current Meter Time Series (W-Mediterranean) \\
\hline & Current Meter Time Series (Adriatic/lonian Sea) \\
\hline Current Meters Time Series (E-Mediterranean) \\
\hline & Lagrangian floats trajectories (W-Mediterranean) \\
\hline & Thermistors time series (W-Mediterranean) \\
\hline & XBT, LXBT, XCTD, Lagrangian drifter profiles (W-Mediterranean) \\
\hline
\end{tabular}




\begin{tabular}{|c|c|}
\hline \multirow[t]{23}{*}{ Bio-chemistry } & Bio-chemical and Physical constituents of settling particles (E-Mediterranean) \\
\hline & Biology in sea water (E-Mediterranean) \\
\hline & Biology in sediment (E-Mediterranean) \\
\hline & Biology in settling particles (E-Mediterranean) \\
\hline & Dissolved biochemical elements (Adriatic/lonian Sea) \\
\hline & Dissolved chemicals in the water column (W-Mediterranean) \\
\hline & Dissolved Chlorofluoro-carbons(Adriatic/lonian Sea) \\
\hline & Dissolved Chlorofluoro-carbons (E-Mediterranean) \\
\hline & Dissolved Gases (Adriatic/lonian Sea) \\
\hline & Dissolved Nutrients and Chlorophyll a(Sicily/Sardinia /Tunisia Region) \\
\hline & Dissolved Nutrients and Organic Carbon(Adriatic/lonian Sea) \\
\hline & Dissolved Nutrients and Organic Carbon in the water column (E-Mediterranean) \\
\hline & Downward fluxes of major biogenic constituents(Adriatic/lonian Sea) \\
\hline & Metals in sediment (E-Mediterranean) \\
\hline & Metals in settling particles (E-Mediterranean) \\
\hline & Metals in Suspended Particulate Matter (E-Mediterranean) \\
\hline & Drifting sediment traps data in the Mediterranean \\
\hline & Moored sediment traps data (W-Mediterranean) \\
\hline & Suspended particulate matter data (W-Mediterranean) \\
\hline & Particulate Matter(Adriatic/lonian Sea) \\
\hline & Phytoplankton (Adriatic/lonian Sea) \\
\hline & Primary Production (Adriatic/lonian Sea) \\
\hline & Primary Production (E-Mediterranean) \\
\hline \multirow[t]{11}{*}{ Specific Data } & Acoustic reflection on marine organisms (W-Mediterranean) \\
\hline & Downward fluxes of major inorganic elements(Adriatic/lonian Sea) \\
\hline & Meteorological data (W-Mediterranean) \\
\hline & Radio-isotopes measurements (W-Mediterranean) \\
\hline & Rare earths measurements (W-Mediterranean) \\
\hline & Sediment core data (W-Mediterranean) \\
\hline & Sediment core major and minor elements(Adriatic/lonian Sea) \\
\hline & Sediment cores analytical data (Adriatic/lonian Sea) \\
\hline & Surface underway data (Adriatic/lonian Sea) \\
\hline & Underwater Irradiance (PAR) in the Mediterranean (Mediterranean) \\
\hline & Underwater Irradiance (PAR) (Adriatic/lonian Sea) \\
\hline
\end{tabular}

Table 1. List of Data Sets Descriptions in MTPII-MATER Catalogue (EDMED)

The above mentioned inventories have been made accessible to the data user community through the World Wide Web (WWW), for each of three particular regions of the Mediterranean Sea [e.g., Western Mediterranean (http://www.ifremer.fr/sismer/program/mater/), Central Mediterranean (http://doga.ogs.trieste.it/mater/), and Eastern Mediterranean (http://hnodc.ncmr.ariadnet.gr/proj mater.html). The Project management unit maintained a similar home page (http://bali.cetiis.fr/mater/) including catalogues, information on the format and quality assurance protocol, interfaces to submit and request data, links to the other servers of the data management network and the EU/MAST "code on data management". The catalogues have been archived in a relational data base management system (RDBMS) under ORACLE . Dynamical links allow up to date publication of the content of the catalogues through WWW during the project implementation. This avoids duplication of work in the WWW server, when updates are made in the RDBMS. At IFREMER data centre, the specific software HTQL (Curé D., 1998) used for the establishment of the above mentioned dynamic links is independent of the RDBMS system itself.

All the EU/MAST-MATER catalogues on WWW have provided a permanent visibility of the status of fieldwork for the overall duration of the project. In addition, the data manager server maintained the up 
to date versions of the management protocol and relevant technical information. Even at the end of the project, the integrated catalogues (in particular concerning the instruments and the data sets descriptions) still reflect the regional implementation of the various tasks of the research project. This was made for practical reasons. Most of the Adriatic data, for example, have been collected on board Italian vessels, using instruments belonging to local research teams. As this information is important to be archived with the data, it remains as it has been transmitted, with links to the corresponding cruise reports. The access to the numerical data themselves can be made from a more integrated approach through the software tools available on the final product (CD-ROM).

\section{Data Sets}

The MTPII-MATER database consists of in-situ observational data. Due to the highly multidisciplinary character of the EU/MAST-MATER Project more than 400 individual numerical parameters were measured in the water column, the suspended matter, the settling particles, the seabed and the pore waters. However, data management activity was mainly focused on only 238 parameters (including the time and space co-ordinates), which have been fully processed, reformatted at the common MEDATLAS format (Maillard C. et al., 2001-a), checked for quality and archived. The later parameters concern the "basic" physical and bio-chemical data, obtained during the project cruises, which are considered as essential data and that need to be shared by all the partners. Additional or specific parameters (e.g., meteorological, biological, etc.) that were occasionally observed, have been marginally archived with quality checks limited to non-missing date and location in the data files, and have been reformatted at the MEDATLAS format, when possible, unless kept at the originator format (Word, Excel, flat ASCII files). The other specific data that have been reported in the cruise reports but non-archived, are either technical or not directly related to the project objectives; they may be obtained only from the data originator. Neither remote sensing data nor model outputs have been integrated in the EU/MAST-MATER final database.

During the project, all basic physical and bio-chemical data sets were actively requested from data originators. Then, they were reformatted at the common format, inventoried in the on-line catalogues and checked for quality according to the protocol described here below (except for the data from cores for which the checks for quality were limited, due to the lack of available methods and software tools). At the end of the data collection phase, the Project co-ordinator and the data managers crosschecked the cruise summary reports and the data received at the data centres, to assure the availability of all the basic data sets collected. The resulting amount of observed data in the MTPII-MATER database is given in Table II, with the number of data files and profiles for each data type and the location of the observations are indicated in Fig. 3 to 6.

The 3788 hydrological profiles (Fig. 3) made by high-resolution CTD-Oxygen-Optical sensors stations, XBT-XCTD and time series from thermistor chains represent a very important input of new available data in the region. Most of them have been made by CTD, but it is noticeable that more than half of the "low resolution" temperature profiles have been made by 7 drifting profilers in the Western Basin (351 stations as weekly ascending profiles from $1200 \mathrm{dbar}$ ). 


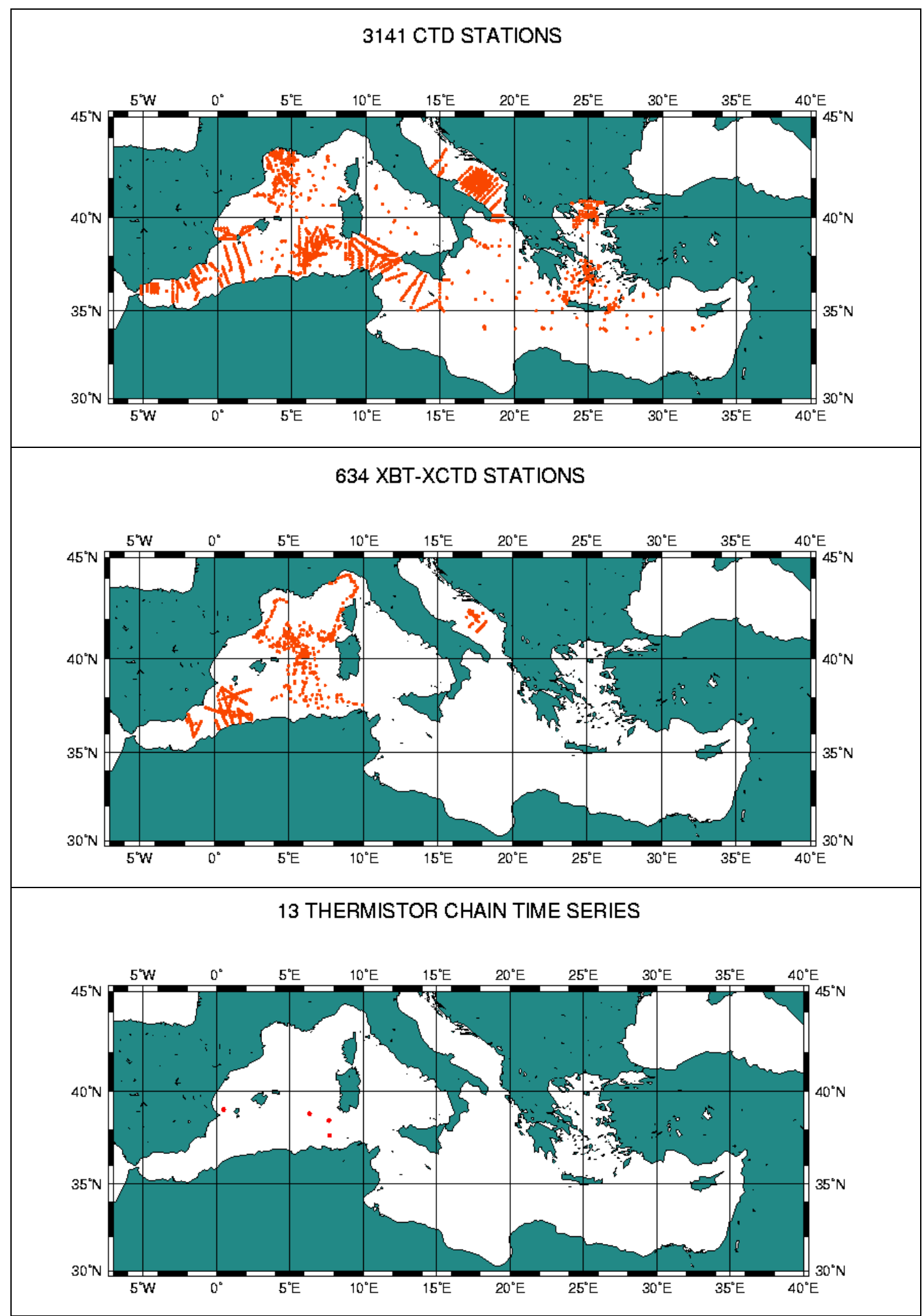

Fig. 3. Position of the Hydrological Measurements

high resolution CTD, XBT and XCTD (including low resolution temperature profiles from drifting profilers), thermistor chains

The release of current data is considerable: 657 long time series from fixed moorings, 38 lagrangian time series in the 0-10 m upper layer, 11 in the 500-600 m layer, 7 at $1200 \mathrm{~m}$ and 6 at $1950 \mathrm{~m}$. In addition to the time series, 360 current vertical profiles have been made in the SW Algerian Basin. Most of these measurements have been made to monitor the Straits (Balearic, Sardinia, Sicily, Otranto, 
Cretan) and the Algerian Current (Fig. 4). The other investigated sites are the Alboran Sea, the Gulf of Lions, the Adriatic Sea and the Aegean but no data have been produced for the Thyrrhenian Sea, and only one site investigated in the Ionian Sea- Levantine Basin.

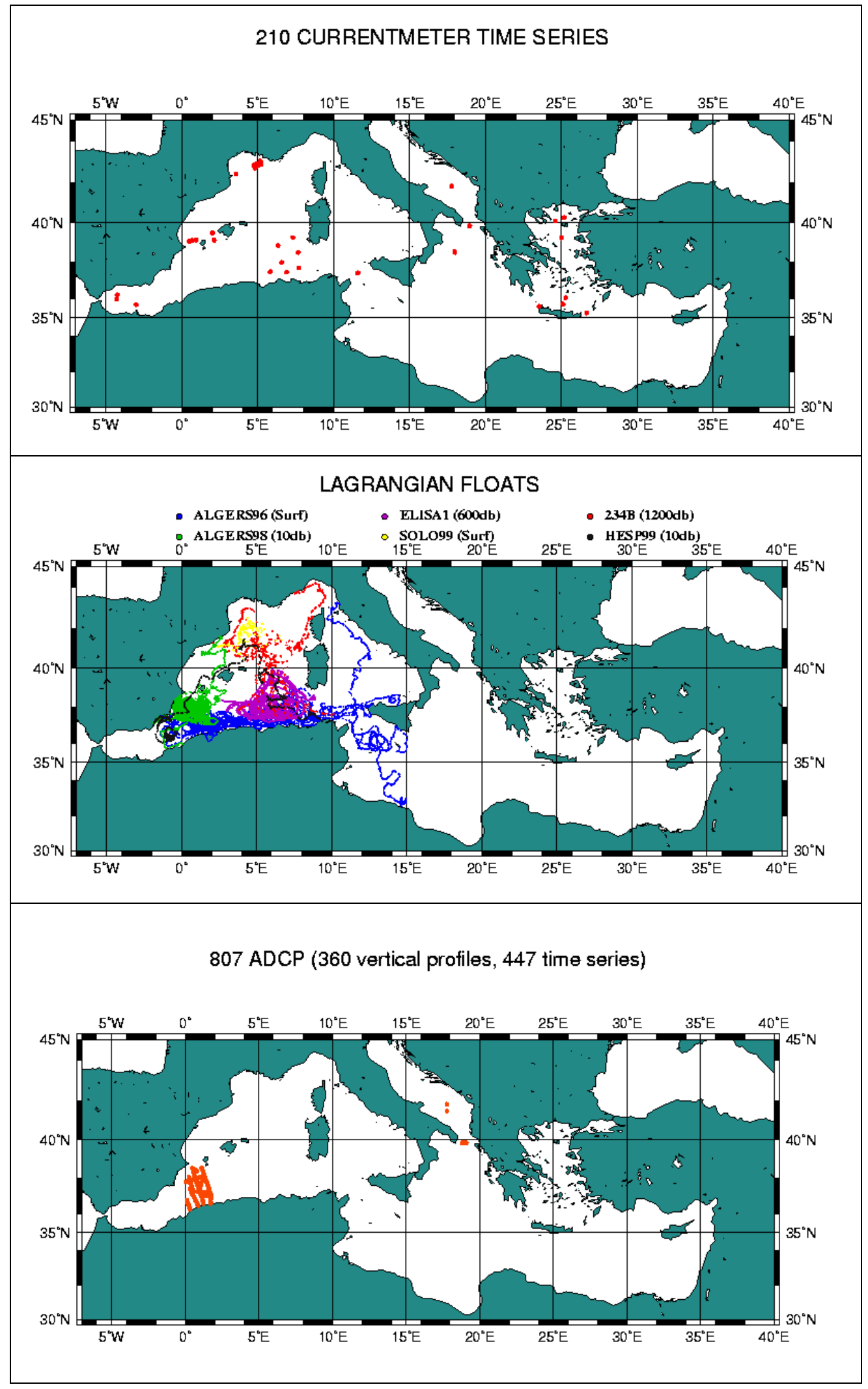

Fig. 4. Position of the Current Measurements 


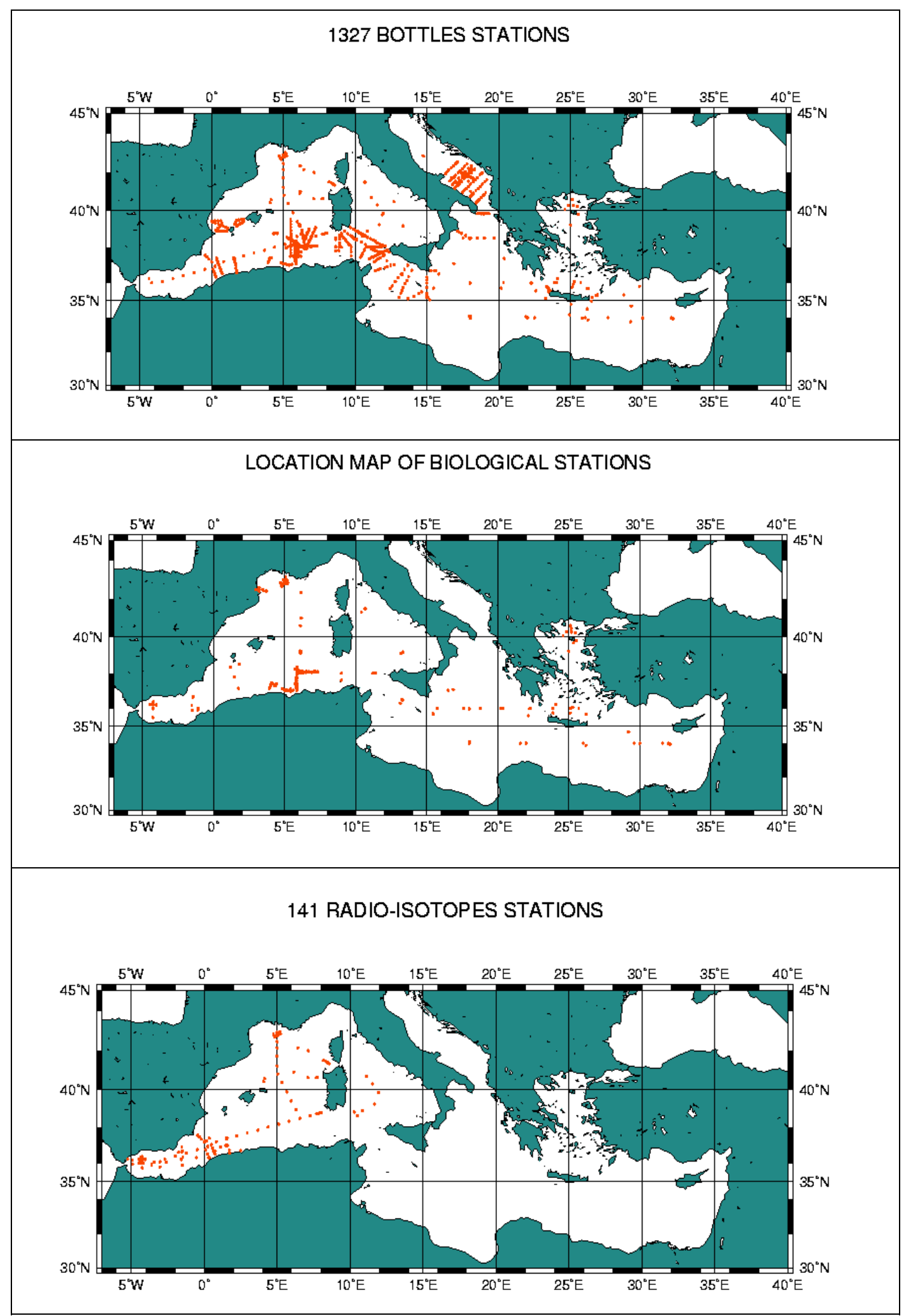

Fig. 5. Positions of the Bio-chemistry Data collected in the water column

The release of bio-chemical data collected in the water column represents 1327 bottle casts with nutrients and various other parameters, 156 microbiology stations, 244 suspended particulate matter and carbon cycle stations, 141 radio-isotopes stations observations. Their distribution (Fig. 5) is denser over the North and West regions, but still this is also an important result of the project as bio-chemical 
data of good quality are not abundant in the historical files. These are the data that have been integrated in the MATER database system; some other biological data are available, but not integrated in the system due to difficulty to organize them in time and space coordinates, or late reception.

Sedimentology data are at the heart of the mass transfer studies and the project released a substantial quantity of these data including 98 sediment trap measurements of settling particles and 70 cores. The sediment traps data are rather well distributed over the whole Mediterranean Sea (Fig. 6), but the core data are limited to the northern sites investigated with current meters. Due to the technical difficulty of integrated the vertical coordinate (from the sea bottom) with the data collected in the water column (from the sea surface), the core data have been formatted at the common format, but the software tools of the MATER database can not be used for the core data.
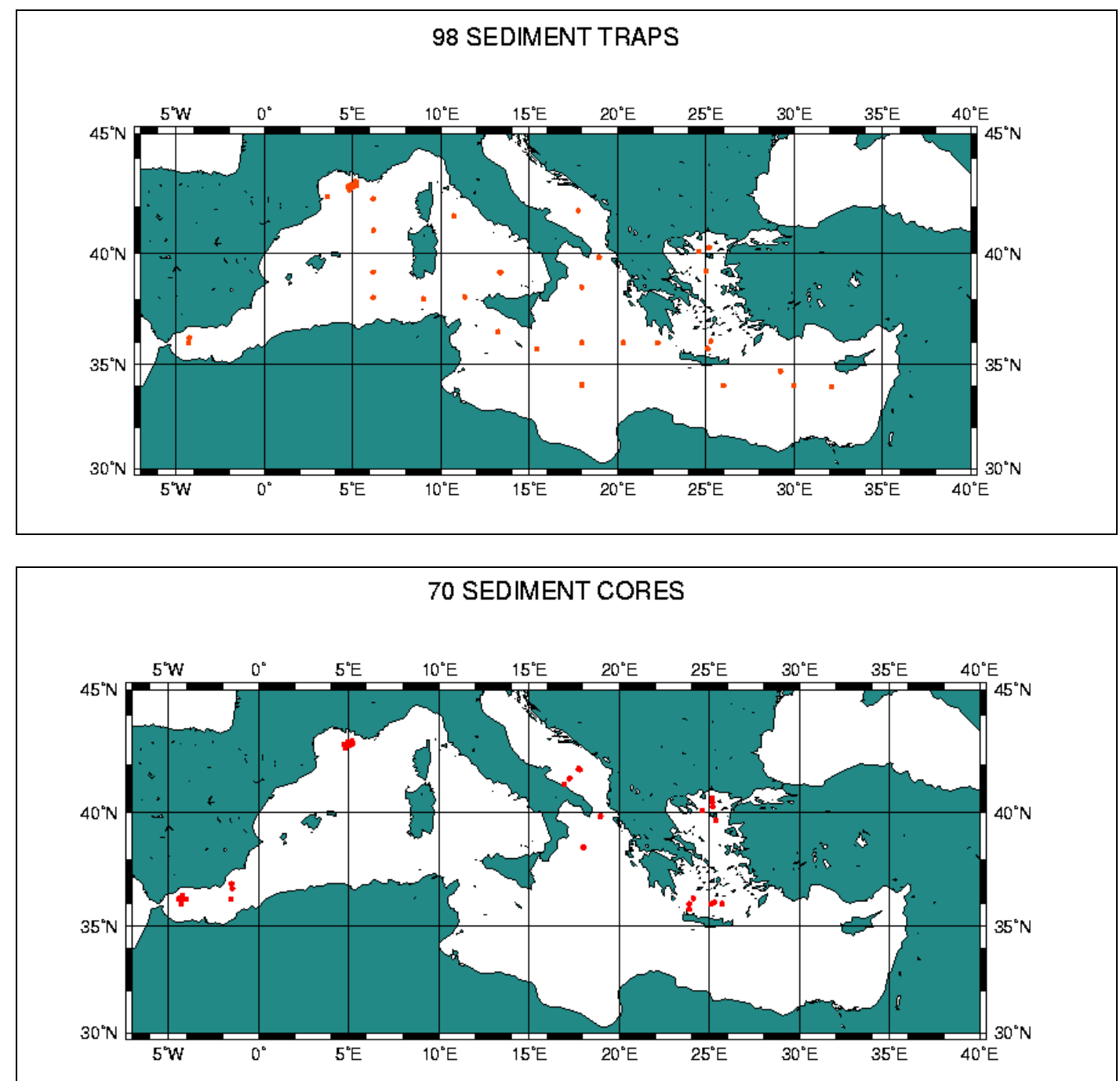

Fig. 6. Positions of biochemistry Data collected in the settling particles near the sediment and in the sediment 


\begin{tabular}{|lccc|}
\hline Data type & Format & Cruise Files profiles \\
\hline CTD & Medatlas & 74 & 3141 \\
XBT, XCTD & Medatlas & 7 & 634 \\
Thermistor chain time series & Medatlas & 7 & 13 \\
Current meter (Eulerian) time series & Medatlas & 108 & 210 \\
Lagrangian Float time series & Medatlas & 6 & 62 \\
ADCP Vertical profiles & Medatlas & 2 & 360 \\
ADCP Time series & Medatlas & 447 & 447 \\
Water Bottle - multi-disciplinary biochemical samples & Medatlas & 65 & 1327 \\
Water Bottle - Biological data (Plankton, Bacteria, Primary Production) & Medatlas & 9 & 156 \\
Water Bottle - Suspended matter & Medatlas & 9 & 244 \\
Sediment trap time series & Medatlas & 45 & 98 \\
Sediment core & Medatlas & 12 \\
Miscellaneous: meteorological data, sediment biochemistry, Acoustic reflection & Medatlas, Word, & 70 \\
on marine organisms, macro- \& meio-fauna, fecal pellets, other specific & Excel, flat ASCll & 67 \\
biological data, isotopes and trace elements in aerosols \& SPM & &
\end{tabular}

Table 2. Number of profiles per data type

\section{Quality Assurance}

Quality assurance (QA) of the data collected during the EU/MAST-MATER Project has been an important element of the overall data management activity. This, included three main tasks: definition of a common protocol for formatting and quality control, implementation of the protocol on all the basic parameters each data centre and finally the validation of the procedures by the Data Quality Experts Group.

The common protocol adopted by the three data centres, is composed by two parts. The first part (Maillard et al., 2001-a) is the protocol for data inventorying (forms), formatting vertical profiles and time series (MEDATLAS format) and quality checking. The second part (Maillard et al., 2001-b) is the data dictionary, with the list of observed parameter codes, names and units. This protocol is based on existing international standards for formatting the data (UNESCO/IOC, 1987), for codes (ICES Service Hydrographique, 2000) and for quality control procedures of the oceanographic data (MAST and UNESCO/IOC, 1993). These reference documents cover only a very limited number of parameters, and it was necessary to extend the procedures to all the basic parameters of MATER, to ensure an overall consistency of units, format and quality checks. Both documents of the MTPII/MATER data management protocol can be retrieved either from the CD-ROM database or from the WWW server.

The format to which the EU/MAST-MATER Project data were formatted is the MEDATLAS format. The MEDATLAS format has been developed for processing hydrological vertical profiles in previous EU/MAST projects (Medatlas Group, 1997). Its development was based on guidelines for formatting and coding data provided by the above mentioned reference documents of the international organizations, such as the IOC/UNESCO and the International Council for the Exploration of the Sea (ICES). The initial MEDATLAS format has been further extended to include more information and to process time series. At its present form the MEDATLAS format is an auto-descriptive ASCIl format, composed of three parts. That are, (a) a cruise header, which is a subset of the cruise/mooring summary report, (b) a station header, which includes latitude, longitude, date and list of parameter codes, names, units and default values, and (c) the observed data, in columns. The first column in the observed data is the vertical coordinate (pressure) for the vertical profiles and the time for the time series. The cruise header appears only once, at the beginning of a file, while the station header and the observations, as many times as 
the number of stations. Different data types of the same cruises are archived in different files. To avoid the wide use of codes, explicit keywords have been defined (e.g., like "DATE", "LATITUDE" etc.). International country codes were included in cruise references and ROSCOP/CSR, and ship codes also. These international codes were extracted from the ICES server (http://www.ices.dk/ocean).

The data dictionary provides a list of the archived parameters along with their GF3 (or extended GF3) (UNESCO/IOC, 1987) codes, their standardised names and units. It also provides some statistics (e.g., minimum and maximum expected values that are used for the quality checks). Standardisation of the data is a prerequisite for merging data sets from different sources. An automatic access requires that the parameter names (or codes) are strictly identical. Coherence implies that the units are identical. All the units are expressed in the International System (IS), or composed with IS units. Exceptions are made for the pressure (in decibar) and the salinity (in Practical Salinity Scale), as commonly used by oceanographers. Parameter codes allow the use of a title of reasonable length to the columns of observations in order to facilitate access of the data. The correspondence between a couple parameter/unit and a code is unique. Some rules have been defined to indicate whether the parameter corresponds to an observation in the water column, the suspended matter or the sediment. The complete data dictionary is available in textual documents down loadable from the CD-ROM and the WWW server; and the reduced list of the basic parameters is directly accessible through WWW.

The Quality Assurance procedure of the data started from the data collection. At first, the source laboratories scientifically validated the various data sets. Then, they were transmitted to one of the three regional data centres where they were reformatted at the common MEDATLAS format, and subjected to additional quality checks (QC), before being safeguarded. As above-mentioned, the MTPII-MATER QC procedure is based on the international recommendations of MAST and UNESCO/IOC that include automatic and visual checks. The QC were performed in three stages: (a) check the format, units, codes and overall completeness and consistency of information (b) check the date and location, and (c) check data points [e.g., minimum/maximum broad range values, search for spikes, stuck sensor, vertical instabilities, comparison with the available climatological statistics from MEDATLAS 1997 (Medatlas Group, 1997) for temperature and salinity and World Ocean Atlas 1994 (Levitus, 1994) for the nutrients, and internal mean and standard deviation for the time series. As a result of the checks, the data are not modified, but a quality flag is added to each numerical value. When any anomaly was met, the source scientist was contacted to validate, correct or eliminate the outlier.

These quality controls were implemented using expert software tools (Bardet D. et al., 2000-a and 2000b) at IFREMER/SISMER and NCMR/HNODC and equivalent tools at OGS. These tools enable automatic checks and display the resulting flags on the screen with editing facilities. The visual checks show the overall consistency of the data within the same data set, find out possible wrong value in case of vertical instability, validate the climatological test for some areas, etc. The results are quality flags added to each numerical value for the location, date and bottom depth (Figure 7), for the data points of vertical profiles (Figure 8-a) and for the data points of the time series (Figure 8-b). Close co-operation between the data managers and the scientists who collected the data greatly contributed to the quality assurance of the data sets. Each quality checked data set was reported to the source scientist who was invited to validate, correct or eliminate any outlier value identified during the quality control.

The procedure and the data dictionary were validated by the Data Quality Group of Experts (DQE) Their advice was in particular valuable in crucial issues related to the selection of the list of basic parameters and also to the validation of the data dictionary for micro-biology and chemicals. 


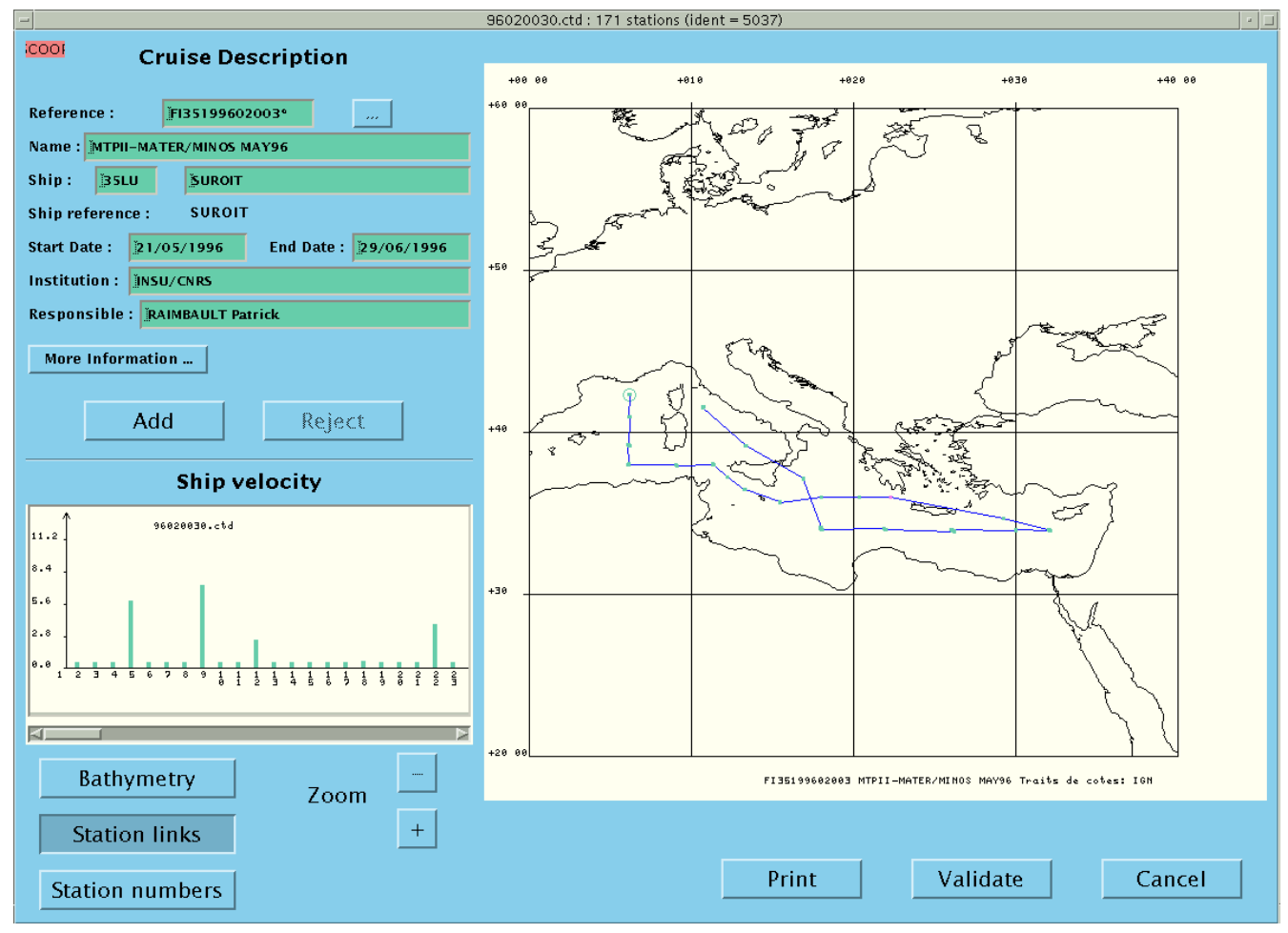

Fig. 7. Check of the Location and Date of the Observations (QC1)

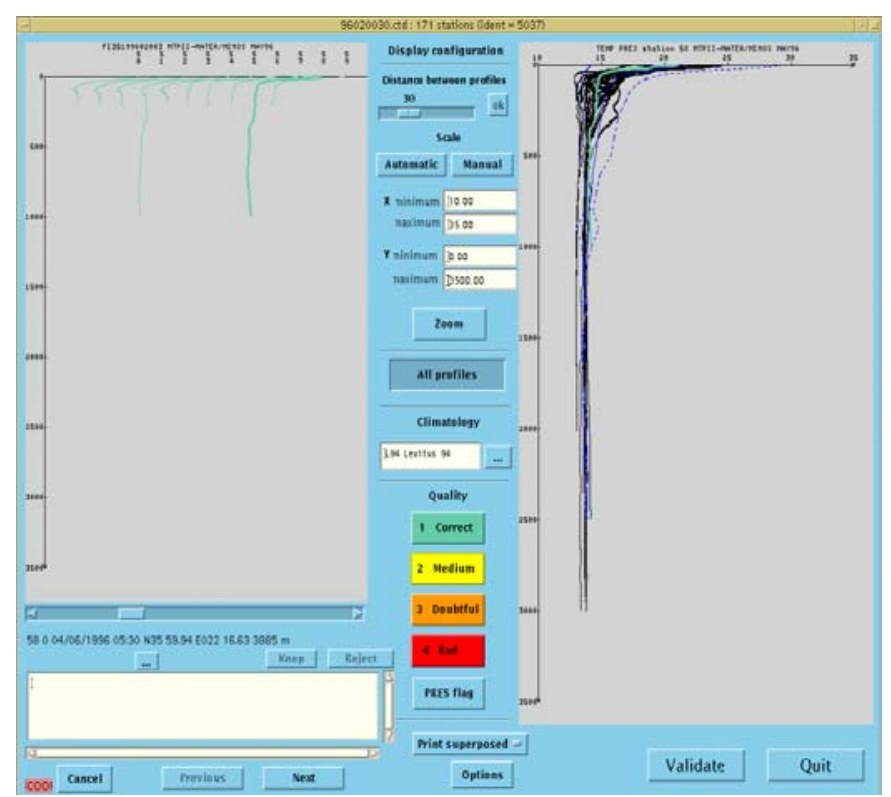

a) vertical profiles

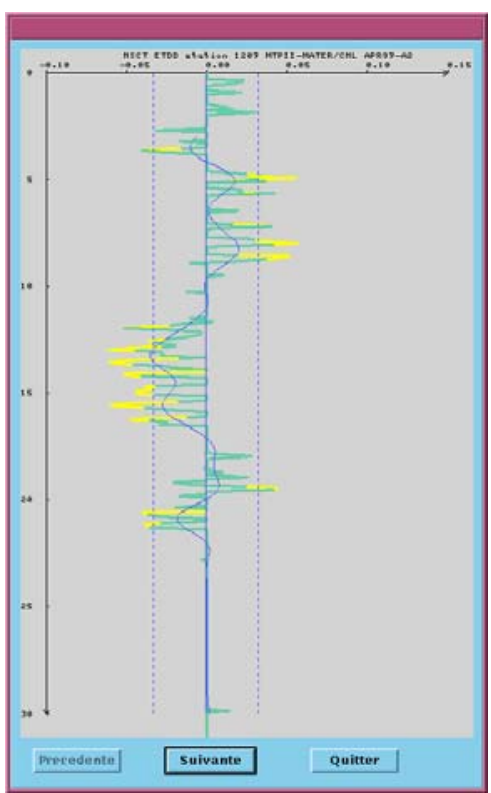

b) Time-series

Fig. 8. Check of the Data Points (QC2)

(in green the current profile, in blue the corresponding estimates climatological variability)

\section{Data Products}

All meta-data (information) and data produced by MTPII--MATER may be accessed through the WWW of the three regional data centres and from an integrated final data product on CD-ROM, which has been prepared to facilitate dissemination of this database among the various users. 
The EU/MAST-MATER database on CD-ROM is an auto-run CD-ROM, which provides two clickable possibilities on the starting window: "Documentation" and "Data Extraction".

The documentation part of the CD-ROM (Figure 9) includes html documents (general information on the project, partnership and data management, all the cruises, moorings, instruments and data sets reports, location maps of the collected data, documentation on format and quality checks), the data files (basic parameters observed data at the MEDATLAS format; other observed data at their original format) and software SELMATER (extraction/visualisation/export of the basic parameters) and Ocean Data View (ODV) software (Schlitzer, 2000 and 2001), which has been developed within the framework of the international "World Ocean Circulation Experiment" (WOCE) programme and made kindly available to MATER by Dr. Reiner Schlitzer (Bremerhaven University). The ODV offers extended visualisation functionalities of the extracted data.

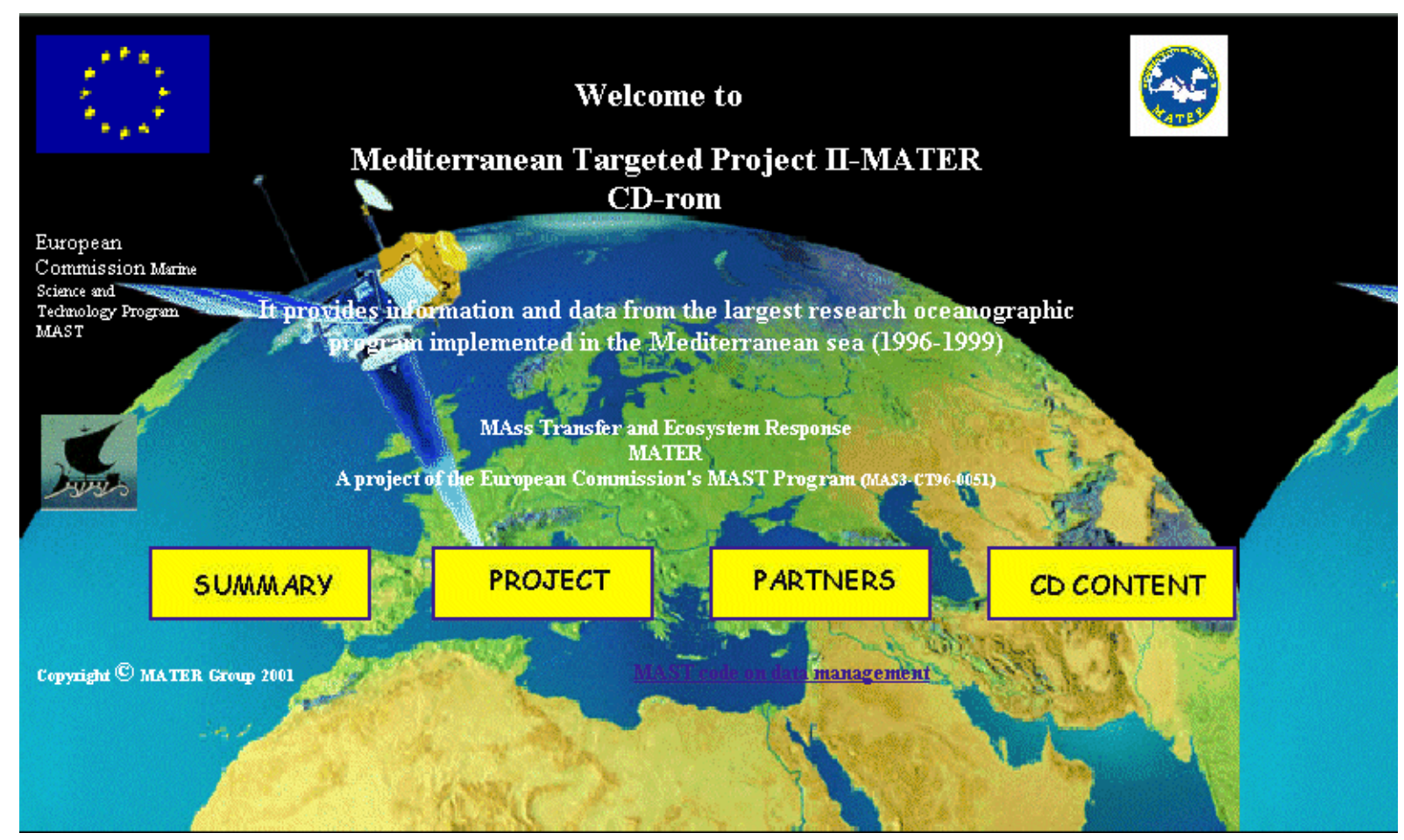

Fig. 9. Home page of the MTPII MATER documentation on CD-ROM

The Data Extraction part of the CD-ROM runs SELMATER and gives a direct access to the basic data and the associated processing tools. This software has been developed by the IFREMER Software Engineering Group ISIB (Larour M., 2000, and Fichaut et al., 2001). The user-friendly SELMATER interface (Figure 10) allows to select data according to multiple selection criteria, such as data type, (Ctd, Xbt, Bottle, Adcp, Current meter, Sediment trap, Thermistor string, Drifting float), parameter (temperature, salinity, phosphate etc..), geographical area, time/period, cruise name, cruise reference, country, ship, quality flag. The data selection can be made with visualisation of the position only or with position and data extraction.

The extracted data can be either observed data, or interpolated data at standard levels defined by the user (for the vertical profiles). For both, three different output formats are available: (a) a fully documented MEDATLAS (ASCII) format, (b) a simple spreadsheet comma-separated values (CSV) format, and (c) ODV compatible export format. 


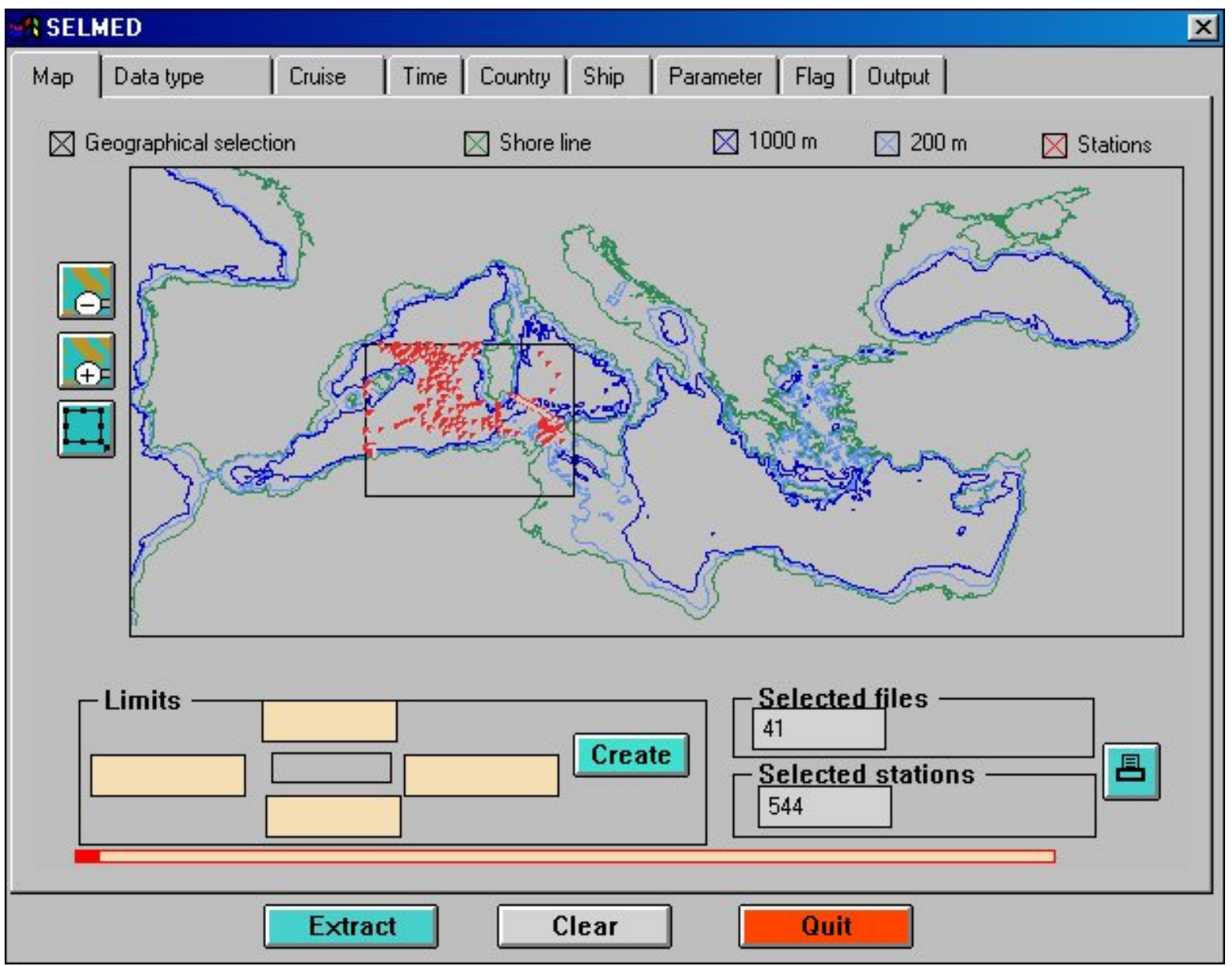

Fig. 10. User interface for data selection

SELMATER allows also simple visualisations of the data from vertical profiles: either any parameter as a function of pressure (the current vertical coordinate in dbar), or a function or any other parameter. One or several cruises can be displayed in superimposed or waterfall plots. Further visualisations (e.g. vertical sections) can be made with the ODV software.

The specific data are available either in MEDATLAS, WORD, EXCEL, flat ASCII files, without further processing tools.

\section{CONCLUSIONS}

A great diversity of oceanographic data types were collected in the Mediterranean Sea, within the framework of the multidisciplinary research project EU/MAST-MATER, during the period 1996-2000. These data not only assisted to the achievement of the project objectives but also will facilitate the proper planning and implementation of further environmental studies in the above-mentioned region.

Data management has been an important component of the EU/MAST/MATER Project. Rather sophisticated methodologies for data formatting and quality checking, as well as, maintenance of up to date catalogues on the WWW servers, enabled the rapid flow of high quality data and information among the participating in the project scientists. Implementation of data management at a regional basis facilitated the close co-operation and exchange of experience among regional data centres and source 
laboratories. Furthermore it enabled the establishment and development of network of data centres, which may be of valuable assistance to the proper planning and implementation of future research activity in the Mediterranean Sea. National mechanisms, responsibilities and computer facilities of the data centres have greatly contributed to the fast publication of the meta-data (cruises, moorings, instruments, data sets) through WWW. In addition, it enabled the proper archive of all the basic physical and bio-chemical parameters, collected during the project, and coherent integration of each data type into the database.

The new, multidisciplinary data set for the Mediterranean Sea along with the data management protocol developed, represent important results of the project, which will be used by scientists and engineers many years beyond the end of the project. The publication of integrated data product on a CD-ROM will facilitate dissemination of the data management results and will be of great assistance to a wide user community (scientists, engineers, policy makers, decision makers, etc.).

\section{Acknowledgments}

This work has been undertaken in the framework of the Mediterranean Targeted Project MTP-IIMATER Project. We acknowledge the support from the European Commission's Marine science and Technology Programme under contract (MAS3-CT96-0051) and of the participating institutes. The authors are very pleased to acknowledge those who were actively involved in the data collection (scientists, technicians, captains and crews of the research vessels), those who carried out the various samples analyses and all those who, by any way, contributed to the development of the EU/MASTMATER database. The authors thank also R. Schlitzer for having kindly made available the WOCE/ODV software to the MATER Group, J.P. Rebert (IRD) for his valuable review and comments and E. Lipiatou (EC) for her constant support all along the project.

\section{References}

Bardet D., Fichaut M., Maudire G., 2000-a. SCOOP: System of Control Oriented Oceanographic Parameters. V4.2 User Manual, IFREMER/SCOOP/MU/110

Bardet D., Fichaut M., Maudire G., 2000-b. SCOOP: System of Control Oriented Oceanographic Parameters. V4.2 Installation Manual, IFREMER/SCOOP/MI/120

Curé D., 1998. HTQL : Interface entre le Web et les bases de données relationnelles, Rap. Int. IFREMER/ DITI/IDT/ISI/98-008

Euronodim Group, 2000. European Directory of Marine Environmental Datasets - EDMED. http://www.sea-search.net/edmed/

Fichaut M., Larour Michel, Maudire G., 2001.SELMATER Data selection from the MATER Database, Rap. Int IFREMER/TMSI/IDM/SISMER-ISIB/SIS01-0042.

ICES Service Hydrographique, 2000. CAUTIONARY NOTE Index server. http://www.ices.dk/ocean/

Larour M., 2000. SELMATER, Specifications, Rap. Int. IFREMER/TMSI/IDM/ISIB 99-01.

Levitus S., 1994. Climatological Atlas of the World Ocean, NOAA Ed., 1994 Edition

Maillard C., Balopoulos, E, Fichaut M., Giorgetti A., Iona A., Latrouite A., Manca B., 2001-a. Mater Data Manual Version 3, Vol 1: Data Management Structure, Inventories, Formats and Codes, Quality Assurance, Rap. Int. IFREMER/TMSI/IDM/SISMER/01-058, 67 pp.

Maillard C., Balopoulos, E, Fichaut M., Giorgetti A., Iona A., Latrouite A., Manca B., 2001-b. Mater Data Manual Version 3, Vol 2: Data Dictionary, Rap. Int. IFREMER/TMSI/IDM/SISMER/01-059, 31 pp.

MAST \& UNESCO/IOC/IODE, 1993. Manual of Quality Control Procedures for Validation of Oceanographic Data. Unesco Manual and Guides 26.436 p. 
Mater Group, 2001. MTPII-MATER Database. Field observations inventories, Data sets and documentation. A project of the European Commission's Marine Science and Technology Programme (MAS3-CT96-0051). Cdrom.

Medatlas Group, 1997. MEDATLAS 1997 Database and climatological atlas of temperature and salinity. 3 Cdroms \& WWW server, IFREMER Ed.

Schlitzer R., Electronic Atlas of WOCE Hydrographic and Tracer Data Now Available, Eos Trans. AGU, 81(5), 45, 2000.

Schlitzer R., Ocean Data View, http://www.awi-bremerhaven.de/GEO/ODV 2001.

UNESCO/IOC 1987, GF3: A general formatting system for geo-referenced data. Vol 2, Technical description of the GF3 format and code tables. UNESCO Manuals and Guides 17. 